\title{
B physics and quarkonia at CMS
}

\author{
Claudia-Elisabeth Wulz ${ }^{1, a}$, on behalf of the CMS Collaboration \\ ${ }^{1}$ Institute of High Energy Physics of the Austrian Academy of Sciences, \\ Nikolsdorfergasse 18, A-1050 Vienna, Austria
}

\begin{abstract}
Selected recent results on B physics and quarkonia are presented. These include a determination of the CP-violating phase $\phi_{s}$ and the decay width difference $\Delta \Gamma_{s}$ of the light and heavy $\mathrm{B}_{s}$ states with $\mathrm{B}_{s} \rightarrow \mathrm{J} / \psi \Phi(1020)$, prompt double-differential production cross sections of $\mathrm{J} / \psi$ and $\psi(2 \mathrm{~S})$, and polarization measurements for $\mathrm{J} / \psi, \psi(2 \mathrm{~S})$, $\Upsilon(1 S), \Upsilon(2 S)$, and $\Upsilon(3 S)$. Results are based on proton-proton data recorded at the LHC at centre-of-mass energies of 7 or $8 \mathrm{TeV}$.
\end{abstract}

\section{Introduction}

Heavy flavours are produced abundantly at the LHC. The CMS experiment [1] is well suited to detect them in spite of large backgrounds, due to its excellent track and vertex reconstruction as well as particle identification capabilities.

For the measurement of the CP-violating weak phase $\phi_{s}$ and the decay width difference $\Delta \Gamma_{s}$ of the light and heavy states of $\mathrm{B}_{s}$ mesons a proton-proton sample of $\mathrm{B}_{s} \rightarrow \mathrm{J} / \psi \Phi\left(\mathrm{K}^{+} \mathrm{K}^{-}\right)$corresponding to an integrated luminosity of about $20 \mathrm{fb}^{-1}$ at a centre-of-mass energy of $\sqrt{s}=8 \mathrm{TeV}$ has been used. A time-dependent and flavour-tagged analysis of the $\mu^{+} \mu^{-} \mathrm{K}^{+} \mathrm{K}^{-}$final state has been performed.

Heavy quarkonia are probes to understand hadron formation. Until recently their production cross sections have only been measured in the lower $p_{T}$ range. Furthermore, polarization measurements by different experiments yielded inconclusive results [2]. CMS has studied quarkonium production and polarization with a data sample of about $4.9 \mathrm{fb}^{-1}$ at $\sqrt{s}=7 \mathrm{TeV}$. The production of S-wave quarkonia has been analyzed using their decays into muon pairs, and P-wave quarkonia production has been analyzed through radiative decays. The CMS measurements presented in this report contribute significantly to the understanding of quarkonia polarization and the underlying theoretical scenarios, in particular in the newly accessible high transverse momentum $\left(p_{T}\right)$ range.

An overview of current B physics results of the CMS experiment may be found here: https://twiki.cern.ch/twiki/bin/view/CMSPublic/PhysicsResultsBPH.

\section{Measurement of $\phi_{s}$ and $\Delta \Gamma_{s}$ of the light and heavy $\mathbf{B}_{s}$ meson states}

The $\mathrm{B}_{s}$ system offers several opportunities to detect possible deviations from the standard model (SM). In this report the measurements of the CP-violating weak phase $\phi_{s}$ and the decay width difference $\Delta \Gamma_{s}$ of the light and heavy $\mathrm{B}_{s}$ mass eigenstates are described. The weak phase $\phi_{s}$ arises from interference

\footnotetext{
a e-mail: claudia.wulz@cern.ch
} 


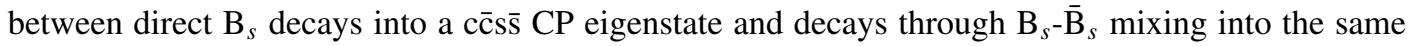
final state. Neglecting penguin diagram contributions, $\phi_{s}$ is related to the CKM matrix elements, with $\phi_{s} \approx-2 \beta_{s}$, and $\beta_{s}=\arg \left(-V_{t s} V_{t b}^{*} / V_{c s} V_{c b}^{*}\right)$. The SM prediction determined from a global fit to experimental data [3] is $-2 \beta_{s}(\mathrm{SM})=0.0363_{-0.0015}^{+0.0016} \mathrm{rad}$. The decay width difference is predicted to be $\Delta \Gamma_{s}=0.087 \pm 0.021 \mathrm{ps}^{-1}$, assuming no new physics in $\mathrm{B}_{s}-\overline{\mathrm{B}}_{s}$ mixing [4].

CMS has performed a time-dependent, flavour-tagged analysis of the $\mathrm{B}_{s} \rightarrow \mathrm{J} / \psi\left(\mu^{+} \mu^{-}\right) \Phi\left(\mathrm{K}^{+} \mathrm{K}^{-}\right)$ final state, with 49000 reconstructed $\mathrm{B}_{s}$ decays at $\sqrt{s}=8 \mathrm{TeV}$ [5]. To disentangle the CP-odd and CP-even final states the set of decay angles $\Theta=\left(\theta_{T}, \varphi_{T}, \psi_{T}\right)$ has been measured. The angles $\theta_{T}$ and $\varphi_{T}$ are the respective polar and azimuthal angles of the $\mu^{+}$in the rest frame of the $\mathrm{J} / \psi$, where the $x$ axis is defined by the decay plane of the $\Phi(1020)$ meson in the $\mathrm{J} / \psi$ rest frame and the $x-y$ plane is defined by the decay plane of the $\Phi \rightarrow \mathrm{K}^{+} \mathrm{K}^{-}$. The helicity angle $\psi_{T}$ is the angle of the $\mathrm{K}^{+}$in the $\Phi$ rest frame with respect to the negative $\mathrm{J} / \psi$ momentum direction.

The differential decay rate of the $\mathrm{B}_{s} \rightarrow \mathrm{J} / \psi \Phi(1020)$ in terms of proper decay length $c t$ and angular variables can be written as [6]:

$$
\frac{d^{4} \Gamma\left(B_{s}(t)\right)}{d \Theta d c t}=\sum_{i=1}^{10} O_{i}(\alpha, c t) \cdot g_{i}(\Theta),
$$

with angular functions $g_{i}\left(\theta_{T}, \varphi_{T}, \psi_{T}\right)$ and time-dependent functions $O_{i}$ :

$$
O_{i}(\alpha, c t)=N_{i} e^{-c t / c \tau}\left[a_{i} \cosh \left(\frac{1}{2} \Delta \Gamma_{s} c t\right)+b_{i} \sinh \left(\frac{1}{2} \Delta \Gamma_{s} c t\right)+c_{i} \cos \left(\Delta m_{s} c t\right)+d_{i} \sin \left(\Delta m_{s} c t\right)\right] .
$$

$\alpha$ is a set of ten physics parameters, including $\phi_{s}$ and $\Delta \Gamma_{s} . b_{i}$ and $d_{i}$ depend on $\phi_{s}$. The definition of the parameters follows LHCb [7].

Events are selected by trigger algorithms optimized to identify $b$ hadrons through their decay to $\mathrm{J} / \psi$ particles originating from secondary vertices. Two muon candidates coming from a common decay vertex are required, each with $p_{T}(\mu)>4 \mathrm{GeV}$, and $p_{T}(\mu \mu)>6.9 \mathrm{GeV}$ with a dimuon mass window between 2.9 and $3.3 \mathrm{GeV}$. The transverse decay length significance $L_{x y} / \sigma\left(L_{x y}\right)$ is required to be greater than three, where $L_{x y}$ is the distance between the primary and secondary vertices in the transverse plane, and $\sigma\left(L_{x y}\right)$ is its uncertainty.

In the offline selection, tighter cuts including a $\mathrm{J} / \psi$ mass constraint $\left|\mathrm{m}_{\mu^{+} \mu^{-}}-\mathrm{M}_{J / \psi}\right|<150 \mathrm{MeV}$ are applied to the muons, and two kaons with momenta greater than $0.7 \mathrm{GeV}$ and a $\Phi$ mass constraint $\left|\mathrm{m}_{K^{+} K^{-}}-\mathrm{M}_{\Phi}\right|<10 \mathrm{MeV}$ are also required. The $\mathrm{B}_{s}(\mu \mu \mathrm{KK})$ is reconstructed by a combined kinematic and vertex fit. The mass of the four-particle state must be between 5.24 and $5.49 \mathrm{GeV}$, and the $\chi^{2}$ vertex fit probability must be greater than $2 \%$. In case of multiple primary vertices the one closest to the $\mathrm{B}_{s}$ is selected.

The main background comes from non-prompt $\mathrm{J} / \psi$ from $\mathrm{b}$ hadrons such as $\mathrm{B}^{0}$ or $\Lambda_{b}$. The lifetime and angular resolution as well as the corresponding measurement efficiencies are determined from simulation. The angular efficiency is modeled by a 3D-function of the decay angles. The angular resolution is not included in the nominal fit, but as a systematic uncertainty. Similarly, the proper decay time efficiency is assumed to be flat in the fitting range from 0.02 to $0.3 \mathrm{~cm}$. Any variations are included as systematic uncertainties. The proper decay time resolution is $70 \mathrm{fs}$ or $21 \mu \mathrm{m}$ if expressed as a length. Fig. 1 shows the distributions of the $\mathrm{J} / \psi \mathrm{KK}$ invariant mass, proper decay length and its uncertainty, respectively.

The flavour of the $\mathrm{B}_{s}$ at production time is determined by tagging the electron or muon from the opposite side $\mathrm{B}$, considering its charge. The tagging performance is optimized by maximizing the tagging power $\mathrm{P}_{t a g}=\epsilon_{\text {tag }}(1-2 \omega)^{2}$ separately for electrons and muons. $\omega$ is the mistag fraction and 

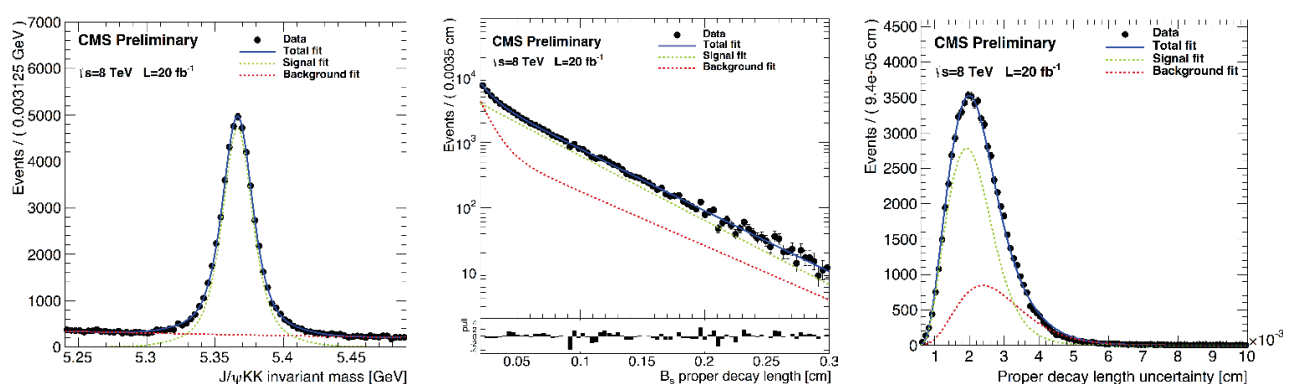

Figure 1. $\mathrm{B}_{s}$ candidate distributions: (Left) invariant mass, (Centre) $\mathrm{B}_{s}$ proper decay length and pull, (Right) $\mathrm{B}_{s}$ proper decay length uncertainty.

$\epsilon_{\text {tag }}$ the tagging efficiency. The tagging power is calibrated with data, using the channel $\mathrm{B}^{+} \rightarrow \mathrm{J} / \psi \mathrm{K}^{+}$, and checked by simulation with $\mathrm{B}^{+} \rightarrow \mathrm{J} / \psi \mathrm{K}^{+}$and $\mathrm{B}_{s} \rightarrow \mathrm{J} / \psi \mathrm{K}^{* 0}$ events. The combined average tagging performance is $\omega=32.3 \pm 0.3 \%, \epsilon_{\text {tag }}=7.67 \pm 0.04 \%$, and $\mathrm{P}_{\text {tag }}=0.97 \pm 0.03 \%$.

An extended maximum likelihood fit to the data, using the signal model defined in eq. (1) and (2) above is used to extract the parameters, constraining $\Delta m_{s}$ to the current world average value of $(17.69 \pm 0.08) \times 10^{12} \hbar / s$ in the fit. Information on the invariant mass, proper decay length, and the three decay angles of the reconstructed $\mathrm{B}_{s}$ candidates is included in the fit. From this multidimensional fit the ten parameters described above are determined. Several sources of systematic uncertainties in the physics parameters are considered by testing the assumptions made in the fit model and those associated in the fit procedure. Biases, which could be intrinsic to the fit model itself, are also taken into account.

Fig. 2 shows the angular distributions of the $\mathrm{B}_{s}$ candidates.
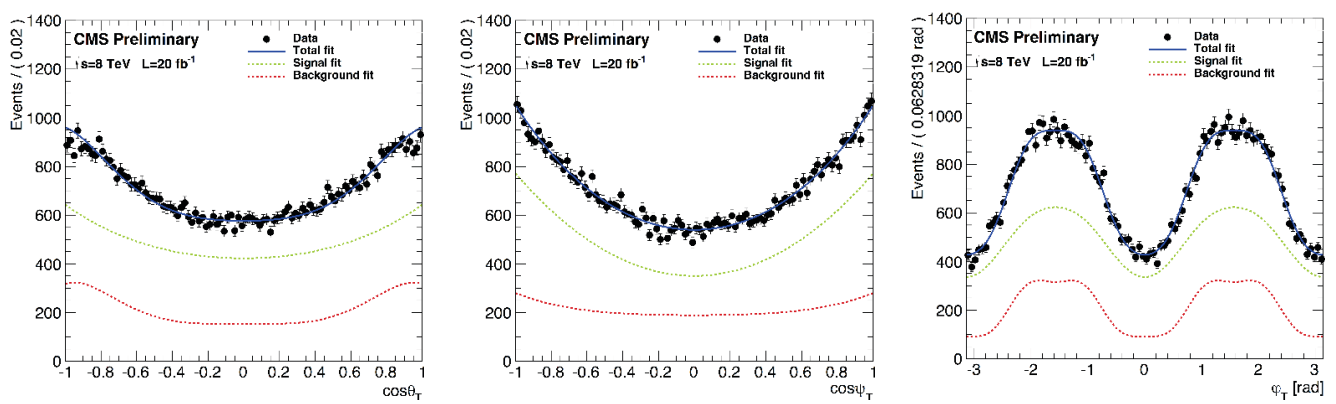

Figure 2. Angular distributions of the $\mathrm{B}_{s}$ candidates. Uncertainties are statistical only.

The measured values for the weak phase and the decay width difference between the $\mathrm{B}_{s}$ mass eigenstates are:

$$
\begin{gathered}
\phi_{s}=-0.03 \pm 0.11 \text { (stat.) } \pm 0.03 \text { (syst.) rad } \\
\Delta \Gamma_{s}=0.096 \pm 0.014 \text { (stat.) } \pm 0.007 \text { (syst.) } \mathrm{ps}^{-1} .
\end{gathered}
$$

The CMS contours in the $\Delta \Gamma_{s}-\phi_{s}$ plane, results from other experiments and experiment combinations performed by the HFAG group [8] are displayed in Fig. 3. 

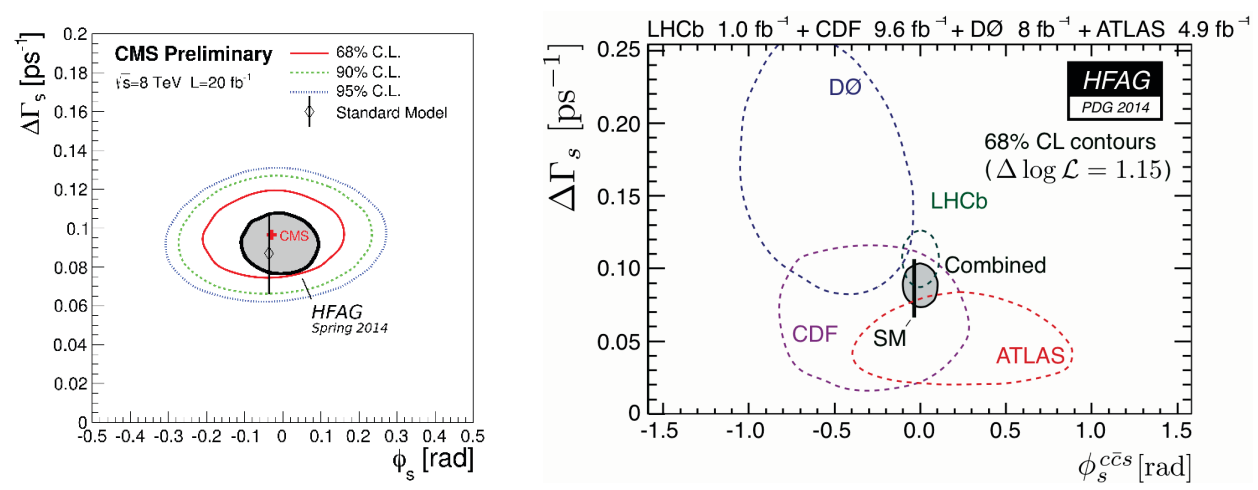

Figure 3. (Left) The $68 \%, 90 \%$ and $95 \%$ C.L. contours in the $\Delta \Gamma_{s}-\phi_{s}$ plane and the SM prediction, (Right) $68 \%$ C.L. contours for different experiments and combination.

\section{Measurement of $\mathbf{J} / \psi$ and $\psi(2 \mathrm{~S})$ prompt double-differential cross sections}

The production of promptly produced $\mathrm{J} / \psi$ and $\psi(2 \mathrm{~S})$ states has been studied using data samples of dimuons recorded at $\sqrt{s}=7 \mathrm{TeV}$, with integrated luminosities of 4.55 and $4.90 \mathrm{fb}^{-1}$, respectively [9]. For the first time it was possible to access the transverse momentum range up to the order of $100 \mathrm{GeV}$. The measurements are based on a two-dimensional analysis of the dimuon invariant mass and pseudo-proper decay length, in the rapidity interval $|y|<1.2$. No distinction was made between directly produced mesons and those originating from the decay of heavier charmonium states. The feed-down to the $\mathrm{J} / \psi$ state from $\psi(2 \mathrm{~S})$ and $\chi_{c}$ decays is about $8 \%$ and $25 \%$, respectively [10].

At the trigger level, opposite-sign dimuons in the invariant mass windows $2.80-3.35 \mathrm{GeV}$ or $3.35-4.05 \mathrm{GeV}$ are selected. The vertex fit $\chi^{2}$ probability must be greater than $0.5 \%$ and the distance to the beam axis smaller than $5 \mathrm{~mm}$. Prompt charmonia are distinguished from B-hadron decays through the dimuon pseudo-proper decay length $\ell=L_{x y} m_{\psi(n S)} / p_{T}$, with $L_{x y}$ being the most probable transverse decay length in the laboratory frame, measured after removing the two muon tracks from the calculated primary vertex position [13]. The prompt charmonium yields are evaluated in different $\left(|y|, p_{T}\right)$ bins. The shape of the mass peaks in the signal region is represented by a Crystal Ball function, whereas the continuum background is described by an exponential function. Fig. 4 shows examples of the projections of the dimuon invariant mass and pseudo-proper decay length from the two-dimensional analysis in a particular $\left(|y|, p_{T}\right)$ bin, for the $\psi(2 \mathrm{~S})$ case.

Systematic uncertainties are evaluated by repeating the two-dimensional fit with different functional forms. They are negligible at low $p_{T}$ and increase to about $2 \%$ for the $\mathrm{J} / \psi$ and $6 \%$ for the $\psi(2 \mathrm{~S})$ in the highest- $p_{T}$ bins. Muon detection efficiencies are measured by a tag-and-probe technique, using event samples recorded with triggers specially designed for this purpose. At high $p_{T}$, the two muons may be emitted close to each other, and the efficiency of the dimuon trigger is different from the product of the two single-muon efficiencies so that it has to be multiplied by a scale factor $\rho$ determined by Monte Carlo simulations.

In Fig. 5 the single-muon detection efficiencies, the $\rho$ parameter, and the acceptances for different polarization scenarios are shown for $\mathrm{J} / \psi$ events. For the cross section measurements an unpolarized scenario has been assumed. This assumption is well justified, as shown in the next section.

Results on the double-differential $\mathrm{J} / \psi$ and $\psi(2 \mathrm{~S})$ production cross sections in various rapidity bins, multiplied by the corresponding branching fractions, are given in Fig. 6. The cross section 
ICNFP 2014
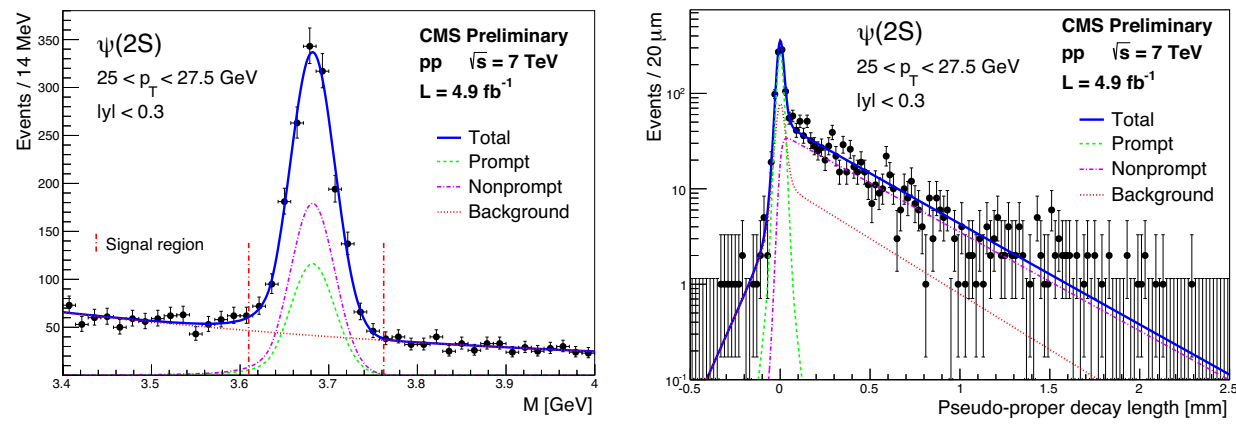

Figure 4. Projections of the (Left) dimuon invariant mass, (Right) pseudo-proper decay length for $\psi(2 S)$ events. The curves represent the results of the fits.
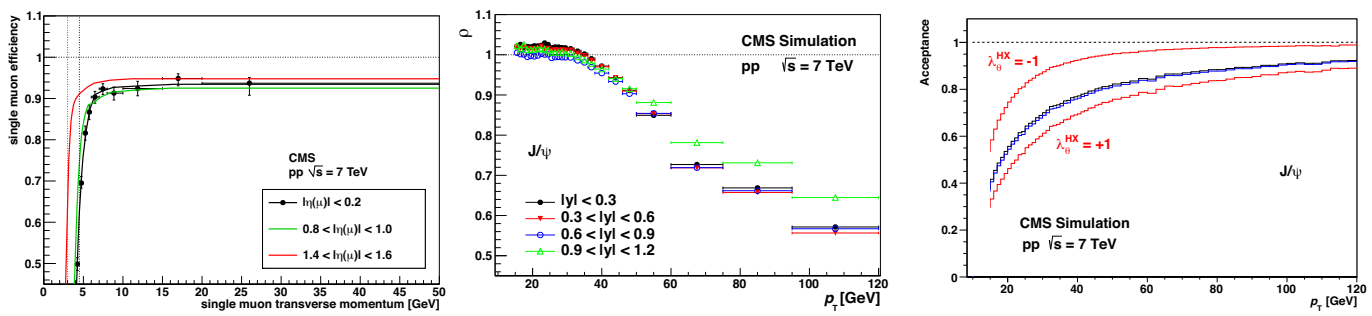

Figure 5. (Left) Single muon detection efficiencies, (Centre) Scale factor $\rho$ reflecting muon correlations, (Right) Acceptances for different polarization scenarios in the centre-of-mass helicity frame.
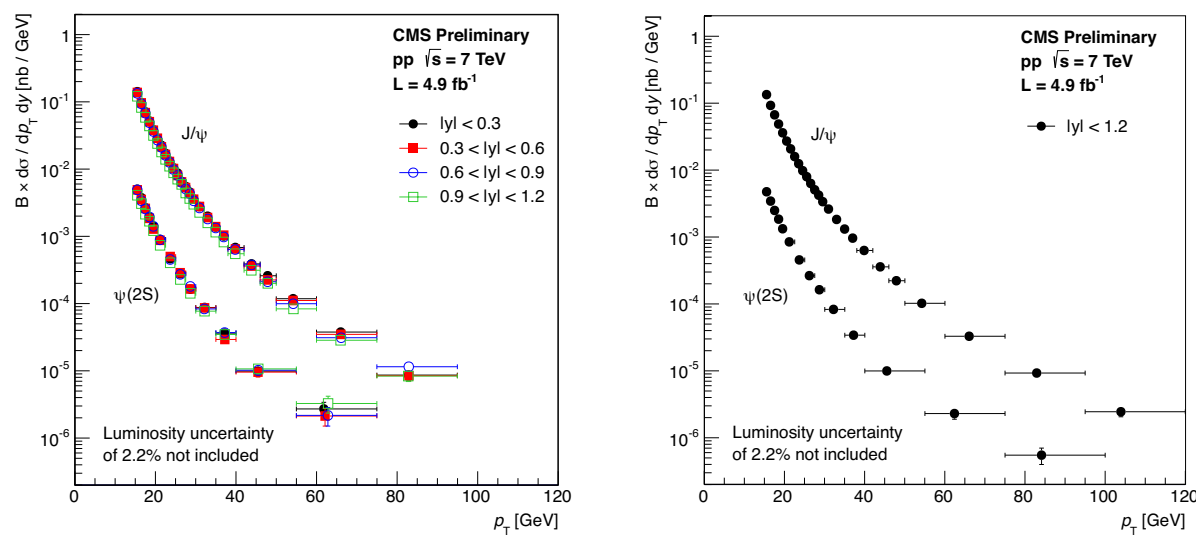

Figure 6. Double-differential cross sections for $\mathrm{J} / \psi$ and $\psi(2 \mathrm{~S})$, as functions of $p_{T}$ in different rapidity regions.

measurement in the broad rapidity range $|y|<1.2$ allows for particularly interesting comparisons with theoretical models in the high- $p_{T}$ range, which has not been probed so far. 


\section{Measurement of the polarization of S- and P-wave quarkonia}

The polarization of $\mathbf{J}^{P C}=1^{--}$vector quarkonium states has been measured through the angular distributions of dimuons from their decay. The most general form of this distribution can be written as [14]

$$
\frac{d N}{d \Omega} \propto \frac{1}{1+3 \lambda_{\vartheta}}\left(1+\lambda_{\vartheta} \cos ^{2} \vartheta+\lambda_{\varphi} \sin ^{2} \vartheta \cos ^{2} 2 \varphi+\lambda_{\vartheta \varphi} \sin 2 \vartheta \cos \varphi\right)
$$

where $\vartheta$ and $\varphi$ are the polar and azimuthal angles, respectively, of the $\mu^{+}$with respect to the chosen polarization frame. The angular distribution parameters $\lambda_{\vartheta}, \lambda_{\varphi}, \lambda_{\vartheta \varphi}$ are different in different polarization frames. A frame-independent parameter is given by $\tilde{\lambda}=\left(\lambda_{\vartheta}+3 \lambda_{\phi}\right) /\left(1-\lambda_{\varphi}\right)$. All these parameters must be measured in order to obtain a reliable measurement of quarkonium polarization.

CMS has measured the polarizations of $\mathrm{J} / \psi, \psi(2 \mathrm{~S})$ [15] and of $\Upsilon(1 S), \Upsilon(2 S), \Upsilon(3 S)[16,17]$ at $\sqrt{s}=7 \mathrm{TeV}$. The full angular decay distributions have been measured in three commonly used polarization frames (Collin-Soper CS, centre-of-mass helicity HX, and perpendicular helicity PX) and frame-independently. Non-prompt charmonium contributions have been removed using pseudoproper decay time measurements. The continuum background is estimated from the side-bands on both sides of the signal regions in the invariant mass distributions such as the ones shown in Fig. 7.
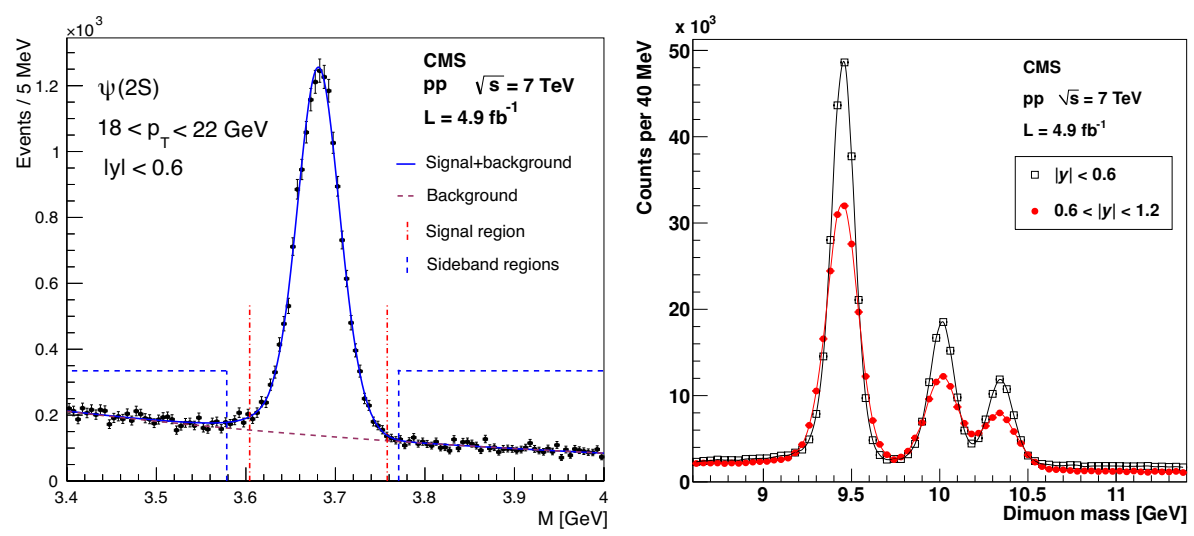

Figure 7. Invariant mass distributions for (Left) $\psi(2 S)$, (Right) $\Upsilon(1 S), \Upsilon(2 S), \Upsilon(3 S)$.

As can be seen from Fig. 8 for $\mathrm{J} / \psi$ and $\psi(2 \mathrm{~S})$, no strong polarization has been found, independently of $p_{T}$ or $y$.
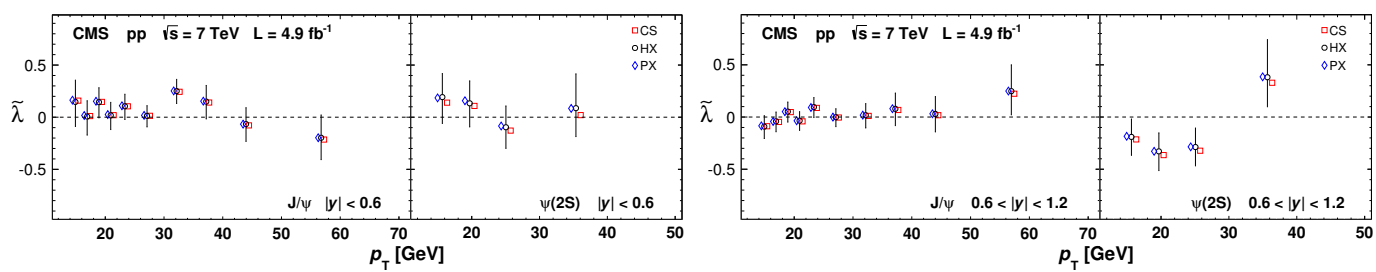

Figure 8. Polarization parameter $\tilde{\lambda}$ as a function of $p_{T}$ for $\mathrm{J} / \psi$ and $\psi(2 \mathrm{~S})$ in different rapidity regions. 
The same is true for $\Upsilon(\mathrm{nS})$, as depicted in Fig. 9, which shows also results from CDF.

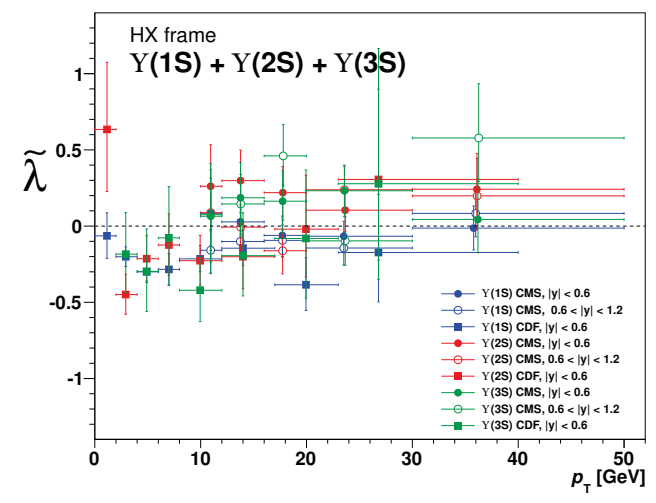

Figure 9. Polarization parameter $\tilde{\lambda}$ as a function of $p_{T}$ for $\Upsilon(1 S), \Upsilon(2 S), \Upsilon(3 S)$ in different rapidity regions, including results from CDF.
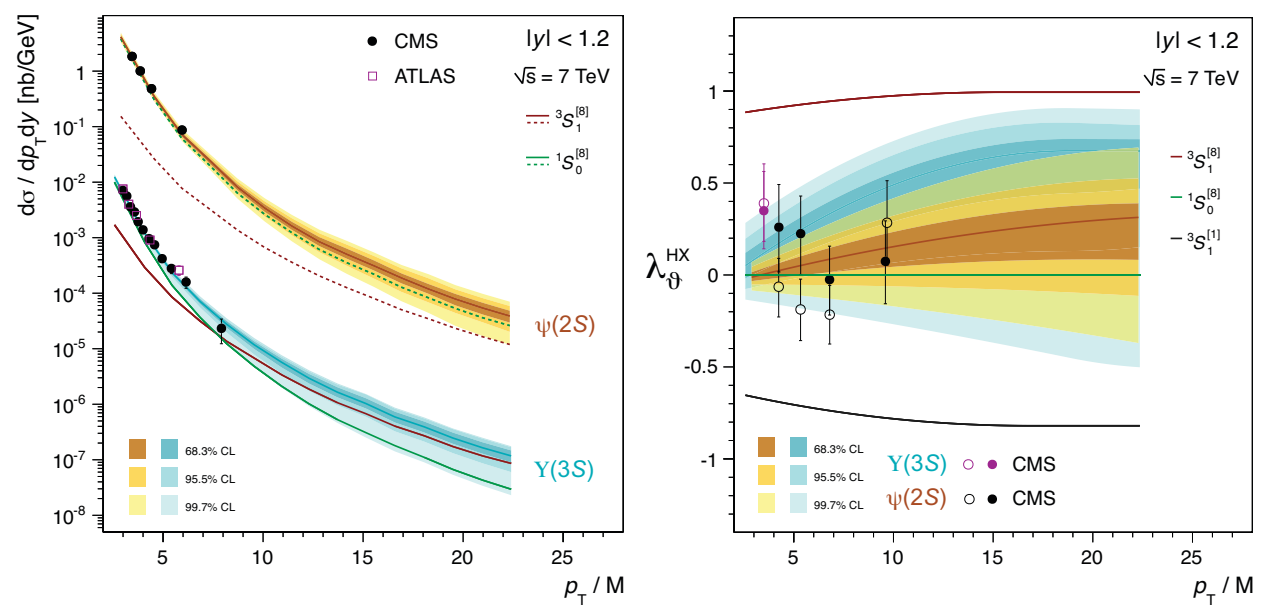

Figure 10. Mass-scaled distributions for $\psi(2 S)$ and $\Upsilon(3 S)$ (Left) diifferential cross sections, (Right) polarization parameter $\lambda_{\vartheta}$ in the HX frame.

A theory for quarkonium production is non-relativistic quantum chromodynamics (NRQCD), an effective field theory that has two factorized steps, the perturbative production of the initial quarkantiquark pair and the non-perturbative hadronization of the quark pair into a bound quarkonium state. NRQCD predicts the existence of intermediate color-octet (CO) states, which subsequently evolve into physical color-singlet (CS) quarkonia by the emission of soft gluons. S-wave vector quarkonia may be formed from heavy quark-antiquark pairs created as $\operatorname{CS}\left({ }^{3} S_{1}^{[1]}\right)$ or one of three color octets $\left({ }^{1} S_{0}^{[8]},{ }^{3} S_{1}^{[8]},{ }^{3} P_{J}^{[8]}\right)$. NRQCD calculations at next-to-leading order (NLO) have been performed and compared with data. 
Fig. 10 shows ATLAS and CMS measurements of the double-differential cross sections and the $\lambda_{\vartheta}$ polarization parameter in the $\mathrm{HX}$ frame as a function of $p_{T}$, mass-rescaled to equalize kinematic effects of different average parton momenta and phase spaces [18]. Extrapolations to higher $p_{T}$ values than the ranges covered by the fitted data are made. Although $\chi_{b}(3 \mathrm{P})$ feed-down contributions to $\Upsilon(3 S)$ are neglected, it can be seen that no strong polarization is present and that the unpolarized ${ }^{1} S_{0}^{[8]}$ component dominates quarkonium production.

\section{Acknowledgments}

I am grateful to my CMS colleagues, in particular Carlos Lourenço, Valentin Knünz, Ilse Krätschmer, and Nuno Viegas Guerreiro Leonardo, for their contributions.

\section{References}

[1] CMS Collaboration, JINST 3, S08004 (2008)

[2] M. Butenschön, B. A. Kniehl, PRL 106, 022003 (2011)

[3] J. Charles et al., PRD 84, 033005 (2011)

[4] A. Lenz and U. Nierste, Proceedings of the 6th International Workshop on the CKM Unitarity Triangle, University of Warwick, UK, 6-10 Sep. 2010, arXiv:1102.4274

[5] CMS Collaboration, CMS-PAS BPH-13-012 (2013)

[6] A. S. Dighe, I. Dunietz, H. J. Lipkin, and J. L. Rosner, PLB 369, 144 (1996)

[7] LHCb Collaboration, PRD 87, 112010 (2013)

[8] http://www.slac.stanford.edu/xorg/hfag/

[9] CMS Collaboration, CMS-PAS BPH-14-001 (2014)

[10] P. Faccioli et al., JHEP 10, 004 (2008)

[11] CMS Collaboration, PLB 727, 381 (2013)

[12] CMS Collaboration, PRL 110, 081802 (2013)

[13] CMS Collaboration, EPJC 71, 1575 (2011)

[14] P. Faccioli et al., EPJC 69, 657 (2010)

[15] CMS Collaboration, PLB 727, 381 (2013)

[16] CMS Collaboration, PRL 108, 151802 (2012)

[17] CMS Collaboration, PRL 110, 081802 (2013)

[18] P. Faccioli et al., PLB 736, 98 (2014) 